\title{
THE FRINGE TRACKING SERVO IN SUSI
}

\author{
PETER R. LAWSON and ANDREW J. BOOTH \\ Chatterton Astronomy Department \\ University of Sydney, NSW 2006, Australia
}

\begin{abstract}
We present a description of the pathlength compensation system used in the Sydney University Stellar Interferometer, and report on the method of fringe tracking that is being implemented. The components of this system are discussed, including the PAPA camera used to detect chromatic fringes, the fringe tracking servo, the delay line and its control.
\end{abstract}

\section{The Sydney University PAPA camera}

A PAPA camera is used in the Sydney University Stellar Interferometer (SUSI) for the observation of group delay fringes in stellar spectra. The camera is a 256 x 256 pixel photon counting array detector operating with speeds upwards of 1 million photons per second and a dead time of $300 \mathrm{~ns}$ for individual photon events. Its principle of operation has been described by Papaliolios et al. (1985). It uses 17 small lenses in an array that provides easy access for optical adjustments, and was optically aligned by the rotation of lenses alone, as described by Lawson (1992).

\section{Laboratory experiments in group delay tracking}

If wavefront tilt is removed from both arms of the interferometer a channeled spectrum can be seen when the light is combined and dispersed. The number of fringes across a fixed bandwidth of a star's spectrum is directly proportional to the pathlength difference between the arriving wavefronts. Short exposure images of stellar spectra can be Fourier transformed and real-time processing used to extract the path difference. Computer simulations and theoretical predictions for this form of tracking have been reported previously by Goodman (1984), Nisenson and Traub (1988), Shao et al. (1988), Buscher (1989), and others.

Here we report preliminary results of laboratory tests of this technique. Figure 1 shows the tracking response of the PAPA camera and processor. A Michelson interferometer was used with a sinusoidal pathlength change of $13 \mu \mathrm{m}$ peak-to-peak at $2.5 \mathrm{~Hz}$ introduced into one arm. Light from a Xenon lamp was used as an artificial star and passed through the interferometer, dispersed, and detected. Approximately 100 photons/frame, over a detected bandwidth of $100 \mathrm{~nm}$, were transformed for the power spectra. The resolution in delay is roughly $2 \mu \mathrm{m}$.

\section{Open-loop performance of the pathlength compensator}

The design of the Optical PathLength Compensator (OPLC) has been outlined previously by Booth et al. (1991) and Gilliand (1992). Presently the OPLC uses a single movable carriage which is microstepped under servo control. The carriage is mounted on rails on a $70 \mathrm{~m}$ long reinforced concrete pier and is monitored by two independent infrared laser metrology systems. HeCd lasers are used with an operating wavelength of $1.15 \mu \mathrm{m}$, and movement errors to an accuracy of $64 \mathrm{~nm}$ optical path difference (OPD) can be tracked. 


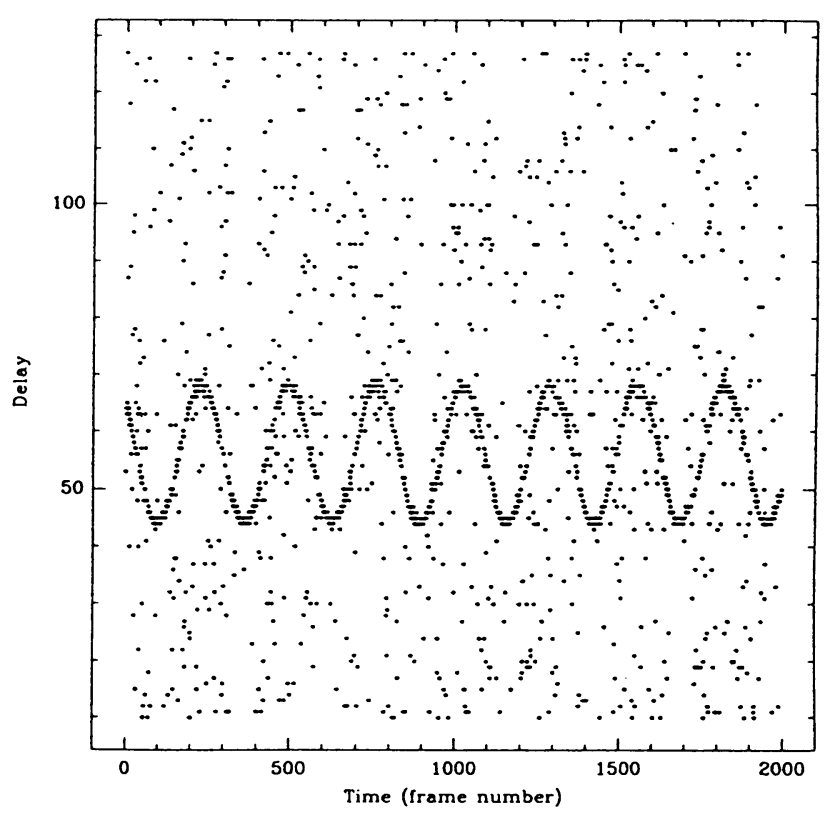

Fig. 1. The tracking response of the PAPA camera and processor during laboratory tests in Sydney. The delay is in units of $0.5 \mu \mathrm{m}$. Each dot indicates the location of the peak in a single (spatial) power spectrum; the sinusoidal changes in delay are obvious, with loss in tracking indicated by the scattered noise. If the pathlength changes have a lower amplitude or frequency then the noise in the tracking disappears altogether.

The path compensation is done in air in a temperature controlled environment and will eventually include correction for longitudinal dispersion, as described by Tango (1990), Large scale path compensation is done differentially using the moving carriage supporting two catseye reflectors pointed in opposite directions along the rails. The total range of adjustment for the fine carriage is $280 \mathrm{~m}$, or $\pm 140 \mathrm{~m}$. The accuracy of the open-loop correction is illustrated in figure 2 .

\section{Current status of control servo}

Preliminary experiments have begun on site with the spectrometer and PAPA camera. For these tests a periscope is used to redirect one of two combined beams from the main optical table to an adjacent table where the camera and spectrometer have been installed. These experiments are being done in parallel with the astronomical program at the shorter baselines.

The monitoring of the metrology system and the guidance of the carriage have in the past been operated from two separate 68000 computers. Presently these two tasks are being combined onto one computer to shorten the servo clock rate to $2 \mathrm{~ms}$ and provide even smoother tracking. 


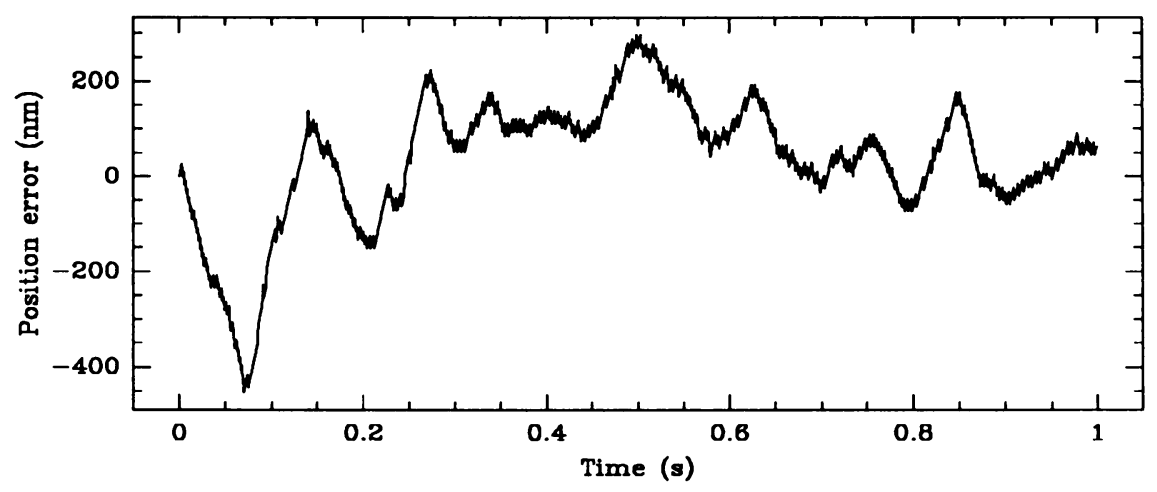

Fig. 2. The tracking smoothness of the carriage. These are for a tracking speed of about $0.1 \mathrm{~mm} / \mathrm{s}$ OPD. The graph of position error shows small oscillations due to the stepper motors. These are caused by DC offsets in the motor drives and can be corrected using the piezos located at the focus of the catseyes.

\section{Acknowledgements}

The SUSI project is funded by the Australian Research Council and the University of Sydney. The support of the Pollock Memorial Fund and the Science Foundation for Physics within the University of Sydney is also gratefully acknowledged. One of the authors (PRL) would also like to acknowledge the support of the a Commonwealth Scholarship and Fellowship Plan through Australian Vice Chancellors' Committee.

\section{References}

C. Papaliolios, P. Nisenson, and S. Ebstein, 'Speckle Imaging with the PAPA camera.' Applied Optics Vol. 24, No. 2, 15 January 1985, 287-292.

P. R. Lawson, 'Artifacts in PAPA camera images.' Submitted to Applied Optics, November 1992.

J. W. Goodman, Statistical Optics. McGraw Hill Book Company: New York 1984, 490-501.

P. Nisenson and W. Traub, 'Magnitude limit of the group delay tracking method for long baseline interferometry' in Interferometric Imaging in Astronomy. National Optical Astronomy Observatories: Tucson AZ, April 1987, 129-133.

M. Shao et. al.,'The Mark III stellar interferometer,' Astron. Astrophys. 193, 1988, 357-371.

D. Buscher, 'Low light level limits to tracking atmospheric fringe wander.' Quantum limited imaging and information processing. 1989 Technical Digest Series Vol. 13, Conference edition. June 12-13 1989, North Falmouth, Cape Cod, MA. Sponsored by O.S.A. and Air Force Office of Scientific Research.

A.J. Booth et al., 'The optical pathlength compensator in SUSI.' Proceedings of the ESO conference on High Resolution Imaging by Interferometry II, European Southern Observatory, October 1991.

Y.A. Gilliand, "Design and Implementation of a Variable Optical Path Length Compensation System for the Sydney University Stellar Interferometer." M.Sc. Thesis, Chatterton Astronomy Department, University of Sydney, July 1992.

W.J. Tango, 'Dispersion in stellar interferometry.' Applied Optics, Vol. 29, No. 4, 1 February 1990. $516-521$. 